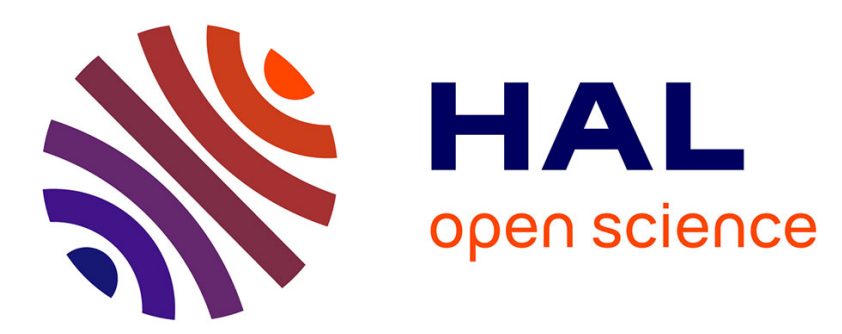

\title{
Nonlocal Poincaré inequalities on Lie groups with polynomial volume growth and Riemannian manifolds
}

\author{
Emmanuel Russ, Yannick Sire
}

\section{To cite this version:}

Emmanuel Russ, Yannick Sire. Nonlocal Poincaré inequalities on Lie groups with polynomial volume growth and Riemannian manifolds. Studia Mathematica, 2011, 203 (2), pp.105-127. 10.4064/sm2032-1. hal-00449531

\author{
HAL Id: hal-00449531 \\ https://hal.science/hal-00449531
}

Submitted on 21 Jan 2010

HAL is a multi-disciplinary open access archive for the deposit and dissemination of scientific research documents, whether they are published or not. The documents may come from teaching and research institutions in France or abroad, or from public or private research centers.
L'archive ouverte pluridisciplinaire HAL, est destinée au dépôt et à la diffusion de documents scientifiques de niveau recherche, publiés ou non, émanant des établissements d'enseignement et de recherche français ou étrangers, des laboratoires publics ou privés. 


\title{
NON LOCAL POINCARÉ INEQUALITIES ON LIE GROUPS WITH POLYNOMIAL VOLUME GROWTH
}

\author{
EMMANUEL RUSS AND YANNICK SIRE
}

\begin{abstract}
Let $G$ be a real connected Lie group with polynomial volume growth, endowed with its Haar measure $d x$. Given a $C^{2}$ positive function $M$ on $G$, we give a sufficient condition for an $L^{2}$ Poincaré inequality with respect to the measure $M(x) d x$ to hold on $G$. We then establish a non-local Poincaré inequality on $G$ with respect to $M(x) d x$.
\end{abstract}

\section{Contents}

1. Introduction

2. A proof of the Poincaré inequality for $d \mu_{M}$

3. Proof of Theorem 1.4

3.1. Rewriting the improved Poincaré inequality

3.2. Off-diagonal $L^{2}$ estimates for the resolvent of $L_{M}$

3.3. Control of $\left\|L_{M}^{\alpha / 4} f\right\|_{L^{2}\left(G, d \mu_{M}\right)}$ and conclusion of the proof of Theorem 1.4

4. Appendix A: Technical lemma

5. Appendix B: Estimates for $g_{j}^{t}$

References

\section{INTRODUCTION}

Let $G$ be a unimodular connected Lie group endowed with a measure $M(x) d x$ where $M \in L^{1}(G)$ and $d x$ stands for the Haar measure on $G$. By "unimodular", we mean that the Haar measure is left and rightinvariant. We always assume that $M=e^{-v}$ where $v$ is a $C^{2}$ function on $G$. If we denote by $\mathcal{G}$ the Lie algebra of $G$, we consider a family

$$
\mathbb{X}=\left\{X_{1}, \ldots, X_{k}\right\}
$$

of left-invariant vector fields on $G$ satisfying the Hörmander condition, i.e. $\mathcal{G}$ is the Lie algebra generated by the $X_{i}^{\prime} s$. A standard metric on $G$, called the Carnot-Caratheodory metric, is naturally associated with $\mathbb{X}$ 
and is defined as follows: let $\ell:[0,1] \rightarrow G$ be an absolutely continuous path. We say that $\ell$ is admissible if there exist measurable functions $a_{1}, \ldots, a_{k}:[0,1] \rightarrow \mathbb{C}$ such that, for almost every $t \in[0,1]$, one has

$$
\ell^{\prime}(t)=\sum_{i=1}^{k} a_{i}(t) X_{i}(\ell(t)) .
$$

If $\ell$ is admissible, its length is defined by

$$
|\ell|=\int_{0}^{1}\left(\sum_{i=1}^{k}\left|a_{i}(t)\right|^{2} d t\right)^{\frac{1}{2}}
$$

For all $x, y \in G$, define $d(x, y)$ as the infimum of the lengths of all admissible paths joining $x$ to $y$ (such a curve exists by the Hörmander condition). This distance is left-invariant. For short, we denote by $|x|$ the distance between $e$, the neutral element of the group and $x$, so that the distance from $x$ to $y$ is equal to $\left|y^{-1} x\right|$.

For all $r>0$, denote by $B(x, r)$ the open ball in $G$ with respect to the Carnot-Caratheodory distance and by $V(r)$ the Haar measure of any ball. There exists $d \in \mathbb{N}^{*}$ (called the local dimension of $(G, \mathbb{X})$ ) and $0<c<C$ such that, for all $r \in(0,1)$,

$$
c r^{d} \leq V(r) \leq C r^{d}
$$

see NSW85. When $r>1$, two situations may occur (see Gui73):

- Either there exist $c, C, D>0$ such that, for all $r>1$,

$$
c r^{D} \leq V(r) \leq C r^{D}
$$

where $D$ is called the dimension at infinity of the group (note that, contrary to $d, D$ does not depend on $\mathbb{X}$ ). The group is said to have polynomial volume growth.

- Or there exist $c_{1}, c_{2}, C_{1}, C_{2}>0$ such that, for all $r>1$,

$$
c_{1} e^{c_{2} r} \leq V(r) \leq C_{1} e^{C_{2} r}
$$

and the group is said to have exponential volume growth.

When $G$ has polynomial volume growth, it is plain to see that there exists $C>0$ such that, for all $r>0$,

$$
V(2 r) \leq C V(r)
$$

which implies that there exist $C>0$ and $\kappa>0$ such that, for all $r>0$ and all $\theta>1$,

$$
V(\theta r) \leq C \theta^{\kappa} V(r)
$$


Denote by $H^{1}\left(G, d \mu_{M}\right)$ the Sobolev space of functions $f \in L^{2}\left(G, d \mu_{M}\right)$ such that $X_{i} f \in L^{2}\left(G, d \mu_{M}\right)$ for all $1 \leq i \leq k$. We are interested in $L^{2}$ Poincaré inequalities for the measure $d \mu_{M}$. In order to state sufficient conditions for such an inequality to hold, we introduce the operator

$$
L_{M} f=-M^{-1} \sum_{i=1}^{k} X_{i}\left\{M X_{i} f\right\}
$$

for all $f$ such that

$f \in \mathcal{D}\left(L_{M}\right):=\left\{g \in H^{1}\left(G, d \mu_{M}\right) ; \frac{1}{\sqrt{M}} X_{i}\left\{M X_{i} f\right\} \in L^{2}(G, d x), \forall 1 \leq i \leq k\right\}$.

One therefore has, for all $f \in \mathcal{D}\left(L_{M}\right)$ and $g \in H^{1}\left(G, d \mu_{M}\right)$,

$$
\int_{G} L_{M} f(x) g(x) d \mu_{M}(x)=\sum_{i=1}^{k} \int_{G} X_{i} f(x) \cdot X_{i} g(x) d \mu_{M}(x) .
$$

In particular, the operator $L_{M}$ is symmetric on $L^{2}\left(G, d \mu_{M}\right)$.

Following [BBCG08], say that a $C^{2}$ function $W: G \rightarrow \mathbb{R}$ is a Lyapunov function if $W(x) \geq 1$ for all $x \in G$ and there exist constants $\theta>0$, $b \geq 0$ and $R>0$ such that, for all $x \in G$,

$$
-L_{M} W(x) \leq-\theta W(x)+b \mathbf{1}_{B(e, R)}(x),
$$

where, for all $A \subset G, \mathbf{1}_{A}$ denotes the characteristic function of $A$. We first claim:

Theorem 1.1. Assume that $G$ is unimodular and that there exists a Lyapunov function $W$ on $G$. Then, $d \mu_{M}$ satisfies the following $L^{2}$ Poincaré inequality: there exists $C>0$ such that, for all function $f \in H^{1}\left(G, d \mu_{M}\right)$ with $\int_{G} f(x) d \mu_{M}(x)=0$,

$$
\int_{G}|f(x)|^{2} d \mu_{M}(x) \leq C \sum_{i=1}^{k} \int_{G}\left|X_{i} f(x)\right|^{2} d \mu_{M}(x) .
$$

Let us give, as a corollary, a sufficient condition on $v$ for (1.4) to hold:

Corollary 1.2. Assume that $G$ is unimodular and there exist constants $a \in(0,1), c>0$ and $R>0$ such that, for all $x \in G$ with $|x|>R$,

$$
a \sum_{i=1}^{k}\left|X_{i} v(x)\right|^{2}-\sum_{i=1}^{k} X_{i}^{2} v(x) \geq c .
$$

Then (1.4) holds. 
Notice that, if (1.5) holds with $a \in\left(0, \frac{1}{2}\right)$, then the Poincaré inequality (1.4) has the following self-improvement:

Proposition 1.3. Assume that $G$ is unimodular and that there exist constants $c>0, R>0$ and $\varepsilon \in(0,1)$ such that, for all $x \in G$,

$$
\frac{1-\varepsilon}{2} \sum_{i=1}^{k}\left|X_{i} v(x)\right|^{2}-\sum_{i=1}^{k} X_{i}^{2} v(x) \geq c \text { whenever }|x|>R .
$$

Then there exists $C>0$ such that, for all function $f \in H^{1}\left(G, d \mu_{M}\right)$ such that $\int_{G} f(x) d \mu_{M}(x)=0$ :

$$
\sum_{i=1}^{k} \int_{G}\left|X_{i} f(x)\right|^{2} d \mu_{M}(x) \geq C \int_{G}|f(x)|^{2}\left(1+\sum_{i=1}^{k}\left|X_{i} v(x)\right|^{2}\right) d \mu_{M}(x)
$$

We finally obtain a Poincaré inequality for $d \mu_{M}$ involving a non local term:

Theorem 1.4. Let $G$ be a unimodular Lie group with polynomial growth. Let $d \mu_{M}=M d x$ be a measure absolutely continuous with respect to the Haar measure on $G$ where $M=e^{-v} \in L^{1}(G)$ and $v \in C^{2}(G)$. Assume that there exist constants $c>0, R>0$ and $\varepsilon \in(0,1)$ such that (1.0) holds. Let $\alpha \in(0,2)$. Then there exists $\lambda_{\alpha}(M)>0$ such that, for any function $f \in \mathcal{D}(G)$ satisfying $\int_{G} f(x) d \mu_{M}(x)=0$,

$$
\begin{array}{r}
\iint_{G \times G} \frac{|f(x)-f(y)|^{2}}{V\left(\left|y^{-1} x\right|\right)\left|y^{-1} x\right|^{\alpha}} d x d \mu_{M}(y) \geq \lambda_{\alpha}(M) \\
\int_{\mathbb{R}^{n}}|f(x)|^{2}\left(1+\sum_{i=1}^{k}\left|X_{i} v(x)\right|^{2}\right) d \mu_{M}(x) .
\end{array}
$$

Note that (1.8) is an improvement of (1.7) in terms of fractional nonlocal quantities. The proof follows the same line as the paper MRS09 but we concentrate here on a more geometric context.

In order to prove Theorem 1.4, we need to introduce fractional powers of $L_{M}$. This is the object of the following developments. Since the operator $L_{M}$ is symmetric and non-negative on $L^{2}\left(G, d \mu_{M}\right)$, we can define the usual power $L^{\beta}$ for any $\beta \in(0,1)$ by means of spectral theory.

Section 2 is devoted to the proof of Theorem 1.1 and Corollary 1.2 . Then, in Section 3, we check $L^{2}$ "off-diagonal" estimates for the resolvent of $L_{M}$ and use them to establish Theorem 1.4. 


\section{A proof of the Poincaré inequality for $d \mu_{M}$}

We follow closely the approach of [BBCG08]. Recall first that the following $L^{2}$ local Poincaré inequality holds on $G$ for the measure $d x$ : for all $R>0$, there exists $C_{R}>0$ such that, for all $x \in G$, all $r \in(0, R)$, all ball $B:=B(x, r)$ and all function $f \in C^{\infty}(B)$,

$$
\int_{B}\left|f(x)-f_{B}\right|^{2} d x \leq C_{R} r^{2} \sum_{i=1}^{k} \int_{B}\left|X_{i} f(x)\right|^{2} d x
$$

where $f_{B}:=\frac{1}{V(r)} \int_{B} f(x) d x$. In the Euclidean context, Poincaré inequalities for vector-fields satisfying Hörmander conditions were obtained by Jerison in Jer86]. A proof of (2.9) in the case of unimodular Lie groups can be found in SC95, but the idea goes back to Var87. A nice survey on this topic can be found in HK00. Notice that no global growth assumption on the volume of balls is required for (2.9) to hold.

The proof of (1.4) relies on the following inequality:

Lemma 2.1. For all function $f \in H^{1}\left(G, d \mu_{M}\right)$ on $G$,

$$
\int_{G} \frac{L_{M} W}{W}(x) f^{2}(x) d \mu_{M}(x) \leq \sum_{i=1}^{k} \int_{G}\left|X_{i} f(x)\right|^{2} d \mu_{M}(x) .
$$

Proof: Assume first that $f$ is compactly supported on $G$. Using the definition of $L_{M}$, one has

$$
\begin{aligned}
\int_{G} \frac{L_{M} W}{W}(x) f^{2}(x) d \mu_{M}(x)= & \sum_{i=1}^{k} \int_{G} X_{i}\left(\frac{f^{2}}{W}\right)(x) \cdot X_{i} W(x) d \mu_{M}(x) \\
= & 2 \sum_{i=1}^{k} \int_{G} \frac{f}{W}(x) X_{i} f(x) \cdot X_{i} W(x) d \mu_{M}(x) \\
& -\sum_{i=1}^{k} \int_{G} \frac{f^{2}}{W^{2}}(x)\left|X_{i} W(x)\right|^{2} d \mu_{M}(x) \\
= & \sum_{i=1}^{k} \int_{G}\left|X_{i} f(x)\right|^{2} d \mu_{M}(x) \\
& -\sum_{i=1}^{k} \int_{G}\left|X_{i} f-\frac{f}{W} X_{i} W\right|^{2}(x) d \mu_{M}(x) \\
\leq & \sum_{i=1}^{k} \int_{G}\left|X_{i} f(x)\right|^{2} d \mu_{M}(x) .
\end{aligned}
$$


Notice that all the previous integrals are finite because of the support condition on $f$. Now, if $f$ is as in Lemma 2.1, consider a nondecreasing sequence of smooth compactly supported functions $\chi_{n}$ satisfying

$$
\mathbf{1}_{B(e, n R)} \leq \chi_{n} \leq 1 \text { and }\left|X_{i} \chi_{n}\right| \leq 1 \text { for all } 1 \leq i \leq k .
$$

Applying (2.10) to $f \chi_{n}$ and letting $n$ go to $+\infty$ yields the desired conclusion, by use of the monotone convergence theorem in the lefthand side and the dominated convergence theorem in the right-hand side.

Let us now establish (1.4). Let $g$ be a smooth function on $G$ and let $f:=g-c$ on $G$ where $c$ is a constant to be chosen. By assumption (1.3),

$$
\int_{G} f^{2}(x) d \mu_{M}(x) \leq \int_{G} f^{2}(x) \frac{L_{M} W}{\theta W}(x) d \mu_{M}(x)+\int_{B(e, R)} f^{2}(x) \frac{b}{\theta W}(x) d \mu_{M}(x) .
$$

Lemma 2.1 shows that (2.10) holds. Let us now turn to the second term in the right-hand side of (2.11). Fix $c$ such that $\int_{B(e, R)} f(x) d \mu_{M}(x)=0$. By (2.9) applied to $f$ on $B(e, R)$ and the fact that $M$ is bounded from above and below on $B(e, R)$, one has

$$
\int_{B(e, R)} f^{2}(x) d \mu_{M}(x) \leq C R^{2} \sum_{i=1}^{k} \int_{B(e, R)}\left|X_{i} f(x)\right|^{2} d \mu_{M}(x)
$$

where the constant $C$ depends on $R$ and $M$. Therefore, using the fact that $W \geq 1$ on $G$,

$$
\int_{B(e, R)} f^{2}(x) \frac{b}{\theta W}(x) d \mu_{M}(x) \leq C R^{2} \sum_{i=1}^{k} \int_{B(e, R)}\left|X_{i} f(x)\right|^{2} d \mu_{M}(x)
$$

where the constant $C$ depends on $R, M, \theta$ and $b$. Gathering (2.11), (2.10) and (2.12) yields

$$
\int_{G}(g(x)-c)^{2} d \mu_{M}(x) \leq C \sum_{i=1}^{k} \int_{G}\left|X_{i} g(x)\right|^{2} d \mu_{M}(x),
$$

which easily implies (1.4) for the function $g$ (and the same dependence for the constant $C$ ).

Proof of Corollary 1.2: according to Theorem 1.1, it is enough to find a Lyapunov function $W$. Define

$$
W(x):=e^{\gamma\left(v(x)-\inf _{G} v\right)}
$$


where $\gamma>0$ will be chosen later. Since

$$
-L_{M} W(x)=\gamma\left(\sum_{i=1}^{k} X_{i}^{2} v(x)-(1-\gamma) \sum_{i=1}^{k}\left|X_{i} v(x)\right|^{2}\right) W(x),
$$

$W$ is a Lyapunov function for $\gamma:=1-a$ because of the assumption on $v$. Indeed, one can take $\theta=c \gamma$ and $b=\max _{B(e, R)}\left\{-L_{M} W+\theta W\right\}$ (recall that $M$ is a $C^{2}$ function).

Let us now prove Proposition 1.3. Observe first that, since $v$ is $C^{2}$ on $G$ and (1.6) holds, there exists $\alpha \in \mathbb{R}$ such that, for all $x \in G$,

$$
\frac{1-\varepsilon}{2} \sum_{i=1}^{k}\left|X_{i} v(x)\right|^{2}-\sum_{i=1}^{k} X_{i}^{2} v(x) \geq \alpha .
$$

Let $f$ be as in the statement of Proposition 1.3 and let $g:=f M^{\frac{1}{2}}$. Since, for all $1 \leq i \leq k$,

$$
X_{i} f=M^{-\frac{1}{2}} X_{i} g-\frac{1}{2} g M^{-\frac{3}{2}} X_{i} M .
$$

Assumption (2.13) yields two positive constants $\beta, \gamma$ such that

$$
\sum_{i=1}^{k} \int_{G}\left|X_{i} f(x)\right|^{2}(x) d \mu_{M}(x)=
$$

$$
\sum_{i=1}^{k} \int_{G}\left(\left|X_{i} g(x)\right|^{2}+\frac{1}{4} g^{2}(x)\left|X_{i} v(x)\right|^{2}+g(x) X_{i} g(x) X_{i} v(x)\right) d x
$$

$$
\begin{gathered}
=\sum_{i=1}^{k} \int_{G}\left(\left|X_{i} g(x)\right|^{2}+\frac{1}{4} g^{2}(x)\left|X_{i} v(x)\right|^{2}+\frac{1}{2} X_{i}\left(g^{2}\right)(x) X_{i} v(x)\right) d x \\
\geq \sum_{i=1}^{k} \int_{G} g^{2}(x)\left(\frac{1}{4}\left|X_{i} v(x)\right|^{2}-\frac{1}{2} X_{i}^{2} v(x)\right) d x \\
\geq \sum_{i=1}^{k} \int_{G} f^{2}(x)\left(\beta\left|X_{i} v(x)\right|^{2}-\gamma\right) d \mu_{M}(x) .
\end{gathered}
$$

The conjunction of (1.4), which holds because of (1.6), and (2.14) yields the desired conclusion.

\section{Proof of Theorem 1.4}

We divide the proof into several steps. 
3.1. Rewriting the improved Poincaré inequality. By the definition of $L_{M}$, the conclusion of Proposition 1.3 means, in terms of operators in $L^{2}\left(G, d \mu_{M}\right)$, that, for some $\lambda>0$,

$$
L_{M} \geq \lambda \mu,
$$

where $\mu$ is the multiplication operator by $1+\sum_{i=1}^{k}\left|X_{i} v\right|^{2}$. Using a functional calculus argument (see [Dav80, p. 110), one deduces from (3.15) that, for any $\alpha \in(0,2)$,

$$
L_{M}^{\alpha / 2} \geq \lambda^{\alpha / 2} \mu^{\alpha / 2}
$$

which implies, thanks to the fact $L_{M}^{\alpha / 2}=\left(L_{M}^{\alpha / 4}\right)^{2}$ and the symmetry of $L_{M}^{\alpha / 4}$ on $L^{2}\left(G, d \mu_{M}\right)$, that

$$
\begin{gathered}
\int_{G}|f(x)|^{2}\left(1+\sum_{i=1}^{k}\left|X_{i} v(x)\right|^{2}\right)^{\alpha / 2} d \mu_{M}(x) \leq \\
C \int_{G}\left|L_{M}^{\alpha / 4} f(x)\right|^{2} d \mu_{M}(x)=C\left\|L_{M}^{\alpha / 4} f\right\|_{L^{2}\left(G, d \mu_{M}\right)}^{2} .
\end{gathered}
$$

The conclusion of Theorem 1.4 will follow by estimating the quantity $\left\|L^{\alpha / 4} f\right\|_{L^{2}\left(G, d \mu_{M}\right)}^{2}$.

3.2. Off-diagonal $L^{2}$ estimates for the resolvent of $L_{M}$. The crucial estimates to derive the desired inequality are some $L^{2}$ "offdiagonal" estimates for the resolvent of $L_{M}$, in the spirit of Gaf59. This is the object of the following lemma.

Lemma 3.1. There exists $C$ with the following property: for all closed disjoint subsets $E, F \subset G$ with $d(E, F)=: d>0$, all function $f \in$ $L^{2}\left(G, d \mu_{M}\right)$ supported in $E$ and all $t>0$,

$$
\begin{gathered}
\left\|\left(I+t L_{M}\right)^{-1} f\right\|_{L^{2}\left(F, d \mu_{M}\right)}+\left\|t L_{M}\left(I+t L_{M}\right)^{-1} f\right\|_{L^{2}\left(F, d \mu_{M}\right)} \leq \\
8 e^{-C \frac{d}{\sqrt{t}}}\|f\|_{L^{2}\left(E, d \mu_{M}\right)} .
\end{gathered}
$$

Proof. We argue as in $\mathrm{AHL}^{+} 02$, Lemma 1.1. From the fact that $L_{M}$ is self-adjoint on $L^{2}\left(G, d \mu_{M}\right)$ we have

$$
\left\|\left(L_{M}-\mu\right)^{-1}\right\|_{L^{2}\left(G, d \mu_{M}\right)} \leq \frac{1}{\operatorname{dist}\left(\mu, \Sigma\left(L_{M}\right)\right)}
$$

where $\Sigma\left(L_{M}\right)$ denotes the spectrum of $L_{M}$, and $\mu \notin \Sigma\left(L_{M}\right)$. Then we deduce that $\left(\mathrm{I}+t L_{M}\right)^{-1}$ is bounded with norm less than 1 for all $t>0$, and it is clearly enough to argue when $0<t<d$. 
In the following computations, we will make explicit the dependence of the measure $d \mu_{M}$ in terms of $M$ for sake of clarity. Define $u_{t}=$ $\left(\mathrm{I}+t L_{M}\right)^{-1} f$, so that, for all function $v \in H^{1}\left(G, d \mu_{M}\right)$,

$$
\begin{array}{r}
\int_{G} u_{t}(x) v(x) M(x) d x+ \\
t \sum_{i=1}^{k} \int_{G} X_{i} u_{t}(x) \cdot X_{i} v(x) M(x) d x= \\
\int_{G} f(x) v(x) M(x) d x .
\end{array}
$$

Fix now a nonnegative function $\eta \in \mathcal{D}(G)$ vanishing on $E$. Since $f$ is supported in $E$, applying (3.16) with $v=\eta^{2} u_{t}$ (remember that $\left.u_{t} \in H^{1}\left(G, d \mu_{M}\right)\right)$ yields

$$
\int_{G} \eta^{2}(x)\left|u_{t}(x)\right|^{2} M(x) d x+t \sum_{i=1}^{k} \int_{G} X_{i} u_{t}(x) \cdot X_{i}\left(\eta^{2} u_{t}\right) M(x) d x=0,
$$

which implies

$$
\begin{gathered}
\int_{G} \eta^{2}(x)\left|u_{t}(x)\right|^{2} M(x) d x+t \int_{G} \eta^{2}(x) \sum_{i=1}^{k}\left|X_{i} u_{t}(x)\right|^{2} M(x) d x \\
=-2 t \sum_{i=1}^{k} \int_{G} \eta(x) u_{t}(x) X_{i} \eta(x) \cdot X_{i} u_{t}(x) M(x) d x \\
\leq t \int_{G}\left|u_{t}(x)\right|^{2} \sum_{i=1}^{k}\left|X_{i} \eta(x)\right|^{2} M(x) d x+ \\
t \int_{G} \eta^{2}(x) \sum_{i=1}^{k}\left|X_{i} u_{t}(x)\right|^{2} M(x) d x
\end{gathered}
$$

hence

$$
\int_{G} \eta^{2}(x)\left|u_{t}(x)\right|^{2} M(x) d x \leq t \int_{G}\left|u_{t}(x)\right|^{2} \sum_{i=1}^{k}\left|X_{i} \eta(x)\right|^{2} M(x) d x .
$$

Let $\zeta$ be a nonnegative smooth function on $G$ such that $\zeta=0$ on $E$, so that $\eta:=e^{\alpha \zeta}-1 \geq 0$ and $\eta$ vanishes on $E$ for some $\alpha>0$ to be chosen. Choosing this particular $\eta$ in (3.17) with $\alpha>0$ gives

$$
\int_{G}\left|e^{\alpha \zeta(x)}-1\right|^{2}\left|u_{t}(x)\right|^{2} M(x) d x \leq
$$




$$
\alpha^{2} t \int_{G}\left|u_{t}(x)\right|^{2} \sum_{i=1}^{k}\left|X_{i} \zeta(x)\right|^{2} e^{2 \alpha \zeta(x)} M(x) d x .
$$

Taking $\alpha=1 /\left(2 \sqrt{t} \max _{i}\left\|X_{i} \zeta\right\|_{\infty}\right)$, one obtains

$$
\int_{G}\left|e^{\alpha \zeta(x)}-1\right|^{2}\left|u_{t}(x)\right|^{2} M(x) d x \leq \frac{1}{4} \int_{G}\left|u_{t}(x)\right|^{2} e^{2 \alpha \zeta(x)} M(x) d x .
$$

Using the fact that the norm of $\left(I+t L_{M}\right)^{-1}$ is bounded by 1 uniformly in $t>0$, this gives

$$
\begin{aligned}
\left\|e^{\alpha \zeta} u_{t}\right\|_{L^{2}\left(G, d \mu_{M}\right)} & \leq\left\|\left(e^{\alpha \zeta}-1\right) u_{t}\right\|_{L^{2}\left(G, d \mu_{M}\right)}+\left\|u_{t}\right\|_{L^{2}\left(G, d \mu_{M}\right)} \\
& \leq \frac{1}{2}\left\|e^{\alpha \zeta} u_{t}\right\|_{L^{2}\left(G, d \mu_{M}\right)}+\|f\|_{L^{2}\left(G, d \mu_{M}\right)},
\end{aligned}
$$

therefore

$$
\int_{G}\left|e^{\alpha \zeta(x)}\right|^{2}\left|u_{t}(x)\right|^{2} M(x) d x \leq 4 \int_{G}|f(x)|^{2} M(x) d x .
$$

We choose now $\zeta$ such that $\zeta=0$ on $E$ as before and additionnally that $\zeta=1$ on $F$. It can furthermore be chosen with $\max _{i=1, \ldots k}\left\|X_{i} \zeta\right\|_{\infty} \leq$ $C / d$, which yields the desired conclusion for the $L^{2}$ norm of $(I+$ $\left.t L_{M}\right)^{-1} f$ with a factor 4 in the right-hand side. Since $t L_{M}\left(\mathrm{I}+t L_{M}\right)^{-1} f=$ $f-\left(\mathrm{I}+t L_{M}\right)^{-1} f$, the desired inequality with a factor 8 readily follows.

\subsection{Control of $\left\|L_{M}^{\alpha / 4} f\right\|_{L^{2}\left(G, d \mu_{M}\right)}$ and conclusion of the proof of}

Theorem 1.4. This is now the heart of the proof to reach the conclusion of Theorem 1.4. The following first lemma is a standard quadratic estimate on powers of subelliptic operators. It is based on spectral theory.

Lemma 3.2. Let $\alpha \in(0,2)$. There exists $C>0$ such that, for all $f \in \mathcal{D}\left(L_{M}\right)$,

$$
\left\|L_{M}^{\alpha / 4} f\right\|_{L^{2}\left(G, d \mu_{M}\right)}^{2} \leq C_{3} \int_{0}^{+\infty} t^{-1-\alpha / 2}\left\|t L_{M}\left(I+t L_{M}\right)^{-1} f\right\|_{L^{2}\left(G, d \mu_{M}\right)}^{2} d t .
$$

We now come to the desired estimate.

Lemma 3.3. Let $\alpha \in(0,2)$. There exists $C>0$ such that, for all $f \in \mathcal{D}(G)$,

$$
\begin{gathered}
\int_{0}^{\infty} t^{-1-\alpha / 2}\left\|t L_{M}\left(I+t L_{M}\right)^{-1} f\right\|_{L^{2}\left(G, d \mu_{M}\right)}^{2} d t \leq \\
C \iint_{G \times G} \frac{|f(x)-f(y)|^{2}}{V\left(\left|y^{-1} x\right|\right)\left|y^{-1} x\right|^{\alpha}} M(x) d x d y .
\end{gathered}
$$


Proof. Fix $t \in(0,+\infty)$. Following Lemma 3.2, we give an upper bound of

$$
\left\|t L_{M}\left(\mathrm{I}+t L_{M}\right)^{-1} f\right\|_{L^{2}\left(G, d \mu_{M}\right)}^{2}
$$

involving first order differences for $f$. Using (1.1), one can pick up a countable family $x_{j}^{t}, j \in \mathbb{N}$, such that the balls $B\left(x_{j}^{t}, \sqrt{t}\right)$ are pairwise disjoint and

$$
G=\bigcup_{j \in \mathbb{N}} B\left(x_{j}^{t}, 2 \sqrt{t}\right)
$$

By Lemma 4.1 in Appendix A, there exists a constant $\widetilde{C}>0$ such that for all $\theta>1$ and all $x \in G$, there are at most $\widetilde{C} \theta^{2 \kappa}$ indexes $j$ such that $\left|x^{-1} x_{j}^{t}\right| \leq \theta \sqrt{t}$ where $\kappa$ is given by (1.2).

For fixed $j$, one has

$$
t L_{M}\left(\mathrm{I}+t L_{M}\right)^{-1} f=t L_{M}\left(\mathrm{I}+t L_{M}\right)^{-1} g^{j, t}
$$

where, for all $x \in G$,

$$
g^{j, t}(x):=f(x)-m^{j, t}
$$

and $m^{j, t}$ is defined by

$$
m^{j, t}:=\frac{1}{V(2 \sqrt{t})} \int_{B\left(x_{j}^{t}, 2 \sqrt{t}\right)} f(y) d y
$$

Note that, here, the mean value of $f$ is computed with respect to the Haar measure on $G$. Since (3.19) holds, one clearly has

$$
\begin{aligned}
\left\|t L_{M}\left(\mathrm{I}+t L_{M}\right)^{-1} f\right\|_{L^{2}\left(G, d \mu_{M}\right)}^{2} & \leq \sum_{j \in \mathbb{N}}\left\|t L_{M}\left(\mathrm{I}+t L_{M}\right)^{-1} f\right\|_{L^{2}\left(B\left(x_{j}^{t}, 2 \sqrt{t}\right), d \mu_{M}\right)}^{2} \\
& =\sum_{j \in \mathbb{N}}\left\|t L_{M}\left(\mathrm{I}+t L_{M}\right)^{-1} g^{j, t}\right\|_{L^{2}\left(B\left(x_{j}^{t}, 2 \sqrt{t}\right), d \mu_{M}\right)}^{2},
\end{aligned}
$$

and we are left with the task of estimating

$$
\left\|t L_{M}\left(\mathrm{I}+t L_{M}\right)^{-1} g^{j, t}\right\|_{L^{2}\left(B\left(x_{j}^{t}, 2 \sqrt{t}\right), d \mu_{M}\right)}^{2} .
$$

To that purpose, set

$$
C_{0}^{j, t}=B\left(x_{j}^{t}, 4 \sqrt{t}\right) \text { and } C_{k}^{j, t}=B\left(x_{j}^{t}, 2^{k+2} \sqrt{t}\right) \backslash B\left(x_{j}^{t}, 2^{k+1} \sqrt{t}\right), \forall k \geq 1 \text {, }
$$

and $g_{k}^{j, t}:=g^{j, t} \mathbf{1}_{C_{k}^{j, t}}, k \geq 0$, where, for any subset $A \subset G, \mathbf{1}_{A}$ is the usual characteristic function of $A$. Since $g^{j, t}=\sum_{k \geq 0} g_{k}^{j, t}$ one has

$$
\begin{gathered}
\left\|t L_{M}\left(\mathrm{I}+t L_{M}\right)^{-1} g^{j, t}\right\|_{L^{2}\left(B\left(x_{j}^{t}, 2 \sqrt{t}\right), d \mu_{M}\right)} \leq \\
\sum_{k \geq 0}\left\|t L_{M}\left(\mathrm{I}+t L_{M}\right)^{-1} g_{k}^{j, t}\right\|_{L^{2}\left(B\left(x_{j}^{t}, 2 \sqrt{t}\right), d \mu_{M}\right)}
\end{gathered}
$$


and, using Lemma 3.1, one obtains (for some constants $C, c>0$ )

$$
\begin{array}{r}
\left\|t L_{M}\left(\mathrm{I}+t L_{M}\right)^{-1} g^{j, t}\right\|_{L^{2}\left(B\left(x_{j}^{t}, 2 \sqrt{t}\right), d \mu_{M}\right)} \leq \\
C\left(\left\|g_{0}^{j, t}\right\|_{L^{2}\left(C_{0}^{j, t}, d \mu_{M}\right)}+\sum_{k \geq 1} e^{-c 2^{k}}\left\|g_{k}^{j, t}\right\|_{L^{2}\left(C_{k}^{j, t}, d \mu_{M}\right)}\right) .
\end{array}
$$

By Cauchy-Schwarz's inequality, we deduce (for another constant $C^{\prime}>$ 0 )

$$
\begin{array}{r}
\left\|t L_{M}\left(\mathrm{I}+t L_{M}\right)^{-1} g^{j, t}\right\|_{L^{2}\left(B\left(x_{j}^{t}, 2 \sqrt{t}\right), d \mu_{M}\right)}^{2} \leq \\
C^{\prime}\left(\left\|g_{0}^{j, t}\right\|_{L^{2}\left(C_{0}^{j, t}, d \mu_{M}\right)}^{2}+\sum_{k \geq 1} e^{-c 2^{k}}\left\|g_{k}^{j, t}\right\|_{L^{2}\left(C_{k}^{j, t}, d \mu_{M}\right)}^{2}\right) .
\end{array}
$$

As a consequence, we have

$$
\begin{aligned}
& \int_{0}^{\infty} t^{-1-\alpha / 2}\left\|t L_{M}\left(\mathrm{I}+t L_{M}\right)^{-1} f\right\|_{L^{2}\left(G, d \mu_{M}\right)}^{2} d t \leq \\
& C^{\prime} \int_{0}^{\infty} t^{-1-\alpha / 2} \sum_{j \geq 0}\left\|g_{0}^{j, t}\right\|_{L^{2}\left(C_{0}^{j, t}, d \mu_{M}\right)}^{2} d t+ \\
& C^{\prime} \int_{0}^{\infty} t^{-1-\alpha / 2} \sum_{k \geq 1} e^{-c 2^{k}} \sum_{j \geq 0}\left\|g_{k}^{j, t}\right\|_{L^{2}\left(C_{k}^{j, t}, d \mu_{M}\right)}^{2} d t .
\end{aligned}
$$

We claim that, and we pospone the proof into Appendix B:

Lemma 3.4. There exists $\bar{C}>0$ such that, for all $t>0$ and all $j \in \mathbb{N}$ :

A. For the first term:

$\left\|g_{0}^{j, t}\right\|_{L^{2}\left(C_{0}^{j, t}, M\right)}^{2} \leq \frac{\bar{C}}{V(\sqrt{t})} \int_{B\left(x_{j}^{t}, 4 \sqrt{t}\right)} \int_{B\left(x_{j}^{t}, 4 \sqrt{t}\right)}|f(x)-f(y)|^{2} d \mu_{M}(x) d y$.

B. For all $k \geq 1$,

$$
\begin{gathered}
\left\|g_{k}^{j, t}\right\|_{L^{2}\left(C_{k}^{j, t}, d \mu_{M}\right)}^{2} \leq \\
\frac{\bar{C}}{V\left(2^{k} \sqrt{t}\right)} \int_{x \in B\left(x_{j}^{t}, 2^{k+2} \sqrt{t}\right)} \int_{y \in B\left(x_{j}^{t}, 2^{k+2 \sqrt{t})}\right.}|f(x)-f(y)|^{2} d \mu_{M}(x) d y .
\end{gathered}
$$


We finish the proof of the theorem. Using Assertion A in Lemma 3.4, summing up on $j \geq 0$ and integrating over $(0, \infty)$, we get

$$
\begin{aligned}
& \int_{0}^{\infty} t^{-1-\alpha / 2} \sum_{j \geq 0}\left\|g_{0}^{j, t}\right\|_{L^{2}\left(C_{0}^{j, t}, d \mu_{M}\right)}^{2} d t=\sum_{j \geq 0} \int_{0}^{\infty} t^{-1-\alpha / 2}\left\|g_{0}^{j, t}\right\|_{L^{2}\left(C_{0}^{j, t}, d \mu_{M}\right)}^{2} d t \\
& \leq \bar{C} \sum_{j \geq 0} \int_{0}^{\infty} \frac{t^{-1-\frac{\alpha}{2}}}{V(\sqrt{t})}\left(\int_{B\left(x_{j}^{t}, 4 \sqrt{t}\right)} \int_{B\left(x_{j}^{t}, 4 \sqrt{t}\right)}|f(x)-f(y)|^{2} d \mu_{M}(x) d y\right) d t \\
& \leq \bar{C} \sum_{j \geq 0} \iint_{(x, y) \in G \times G}|f(x)-f(y)|^{2} M(x) \times \\
& \left(\int_{t \geq \max }\left\{\frac{\left|x^{-1} x_{j}^{t}\right|^{2}}{16} ; \frac{\left|y^{-1} x_{j}^{t}\right|^{2}}{16}\right\} \frac{t^{-1-\frac{\alpha}{2}}}{V(\sqrt{t})} d t\right) d x d y .
\end{aligned}
$$

The Fubini theorem now shows

$$
\begin{aligned}
& \sum_{j \geq 0} \int_{t \geq \max }\left\{\frac{\left|x^{-1} x_{j}^{t}\right|^{2}}{16} ; \frac{\left|y^{-1} x_{j}^{t}\right|^{2}}{16}\right\} \frac{t^{-1-\frac{\alpha}{2}}}{V(\sqrt{t})} d t= \\
& \int_{0}^{\infty} \frac{t^{-1-\frac{\alpha}{2}}}{V(\sqrt{t})} \sum_{j \geq 0} \mathbf{1}\left(\max \left\{\frac{\left|x^{-1} x_{j}^{t}\right|^{2}}{16} ; \frac{\left|y^{-1} x_{j}^{t}\right|^{2}}{16}\right\},+\infty\right)^{(t) d t .}
\end{aligned}
$$

Observe that, by Lemma 4.1, there is a constant $N \in \mathbb{N}$ such that, for all $t>0$, there are at most $N$ indexes $j$ such that $\left|x^{-1} x_{j}^{t}\right|^{2}<16 t$ and $\left|y^{-1} x_{j}^{t}\right|^{2}<16 t$, and for these indexes $j$, one has $\left|x^{-1} y\right|<8 \sqrt{t}$. It therefore follows that

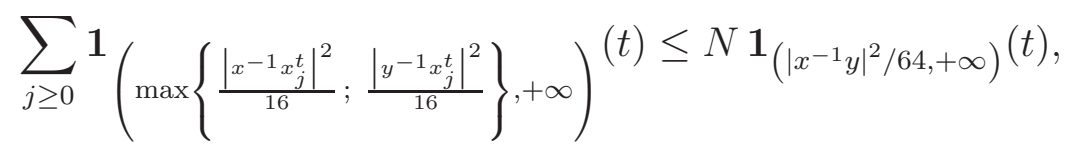

so that, by (1.1),

$$
\begin{aligned}
& \int_{0}^{\infty} t^{-1-\alpha / 2} \sum_{j}\left\|g_{0}^{j, t}\right\|_{L^{2}\left(C_{0}^{j, t}, d \mu_{M}\right)}^{2} d t \\
& \leq \bar{C} N \iint_{G \times G}|f(x)-f(y)|^{2} M(x)\left(\int_{\left|x^{-1} y\right|^{2} / 64}^{\infty} \frac{t^{-1-\frac{\alpha}{2}}}{V(\sqrt{t})} d t\right) d x d y \\
& \leq \bar{C} N \iint_{G \times G} \frac{|f(x)-f(y)|^{2}}{V\left(\left|x^{-1} y\right|\right)\left|x^{-1} y\right|^{\alpha}} d \mu_{M}(x) d y .
\end{aligned}
$$

Using now Assertion B in Lemma 3.4, we obtain, for all $j \geq 0$ and all $k \geq 1$, 


$$
\begin{aligned}
& \int_{0}^{\infty} t^{-1-\alpha / 2} \sum_{j \geq 0}\left\|g_{k}^{j, t}\right\|_{2}^{2} d t \\
& \leq \bar{C} \sum_{j \geq 0} \int_{0}^{\infty} \frac{t^{-1-\frac{\alpha}{2}}}{V\left(2^{k} \sqrt{t}\right)}\left(\iint_{B\left(x_{j}^{t}, 2^{k+2} \sqrt{t}\right) \times B\left(x_{j}^{t}, 2^{k+2} \sqrt{t}\right)}|f(x)-f(y)|^{2} M(x) d x d y\right) d t \\
& \leq \bar{C} \sum_{j \geq 0} \iint_{x, y \in G}|f(x)-f(y)|^{2} M(x) \times \\
& \left(\int_{0}^{\infty} \frac{t^{-1-\frac{\alpha}{2}}}{V\left(2^{k} \sqrt{t}\right)} \mathbf{1}\left(\max \left\{\frac{\left|x^{-1} x_{j}^{t}\right|^{2}}{4^{k+2}}, \frac{\left|y^{-1} x_{j}^{t}\right|^{2}}{4^{k+2}}\right\},+\infty\right) d t\right) d x d y .
\end{aligned}
$$

But, given $t>0, x, y \in G$, by Lemma 4.1 again, there exist at most $\widetilde{C} 2^{2 k \kappa}$ indexes $j$ such that

$$
\left|x^{-1} x_{j}^{t}\right| \leq 2^{k+2} \sqrt{t} \text { and }\left|y^{-1} x_{j}^{t}\right| \leq 2^{k+2} \sqrt{t}
$$

and for these indexes $j,\left|x^{-1} y\right| \leq 2^{k+3} \sqrt{t}$. As a consequence,

$$
\begin{aligned}
& \int_{0}^{\infty} \frac{t^{-1-\frac{\alpha}{2}}}{V\left(2^{k} \sqrt{t}\right)} \sum_{j \geq 0} \mathbf{1}\left(\max \left\{\frac{\left|x^{-1} x_{j}^{t}\right|^{2}}{4^{k+2}}, \frac{\left|x^{-1} x_{j}^{t}\right|^{2}}{4^{k+2}}\right\},+\infty\right) \\
& \widetilde{C} 2^{2 k \kappa} \int_{t \geq \frac{\left|x^{-1} y\right|^{2}}{4^{k+3}}} \frac{t^{-1-\frac{\alpha}{2}}}{V\left(2^{k} \sqrt{t}\right)} d t \leq \\
& \widetilde{C}^{\prime} \frac{2^{k \kappa+\alpha)}}{V\left(\left|x^{-1} y\right|\right)\left|x^{-1} y\right|^{\alpha}},
\end{aligned}
$$

for some other constant $\widetilde{C}^{\prime}>0$, and therefore

$$
\begin{gathered}
\int_{0}^{\infty} \frac{t^{-1-\alpha / 2}}{V\left(2^{k} \sqrt{t}\right)} \sum_{j}\left\|g_{k}^{j, t}\right\|_{L^{2}\left(C_{0}^{j, t}, d \mu_{M}\right)}^{2} d t \leq \\
\bar{C} \widetilde{C}^{\prime} 2^{k(2 \kappa+\alpha)} \iint_{G \times G} \frac{|f(x)-f(y)|^{2}}{V\left(\left|x^{-1} y\right|\right)\left|x^{-1} y\right|^{\alpha}} M(x) d x d y .
\end{gathered}
$$


We can now conclude the proof of Lemma 3.3, using Lemma 3.2, (3.21), (3.24) and (3.25). We have proved, by reconsidering (3.23):

$$
\begin{gathered}
\int_{0}^{\infty} t^{-1-\alpha / 2}\left\|t L_{M}\left(\mathrm{I}+t L_{M}\right)^{-1} f\right\|_{L^{2}\left(G, d \mu_{M}\right)}^{2} d t \leq \\
C^{\prime} \bar{C} N \iint_{G \times G} \frac{|f(x)-f(y)|^{2}}{V\left(\left|x^{-1} y\right|\right)|x-y|^{\alpha}} M(x) d x d y \\
+\sum_{k \geq 1} C^{\prime} \bar{C} \widetilde{C}^{\prime} 2^{k(2 \kappa+\alpha)} e^{-c 2^{k}} \iint_{G \times G} \frac{|f(x)-f(y)|^{2}}{V\left(\left|x^{-1} y\right|\right)\left|x^{-1} y\right|^{\alpha}} M(x) d x d y
\end{gathered}
$$

and we deduce that

$$
\begin{gathered}
\int_{0}^{\infty} t^{-1-\alpha / 2}\left\|t L_{M}\left(\mathrm{I}+t L_{M}\right)^{-1} f\right\|_{L^{2}\left(G, d \mu_{M}\right)}^{2} d t \leq \\
C \iint_{G \times G} \frac{|f(x)-f(y)|^{2}}{V\left(\left|x^{-1} y\right|\right)\left|x^{-1} y\right|^{\alpha}} d \mu_{M}(x) d y
\end{gathered}
$$

for some constant $C$ as claimed in the statement.

Remark 3.5. In the Euclidean context, Strichartz proved in (Str67) that, when $0<\alpha<2$, for all $p \in(1,+\infty)$,

$$
\left\|(-\Delta)^{\alpha / 4} f\right\|_{L^{p}\left(\mathbb{R}^{n}\right)} \leq C_{\alpha, p}\left\|S_{\alpha} f\right\|_{L^{p}\left(\mathbb{R}^{n}\right)}
$$

where

$$
S_{\alpha} f(x)=\left(\int_{0}^{+\infty}\left(\int_{B}|f(x+r y)-f(x)| d y\right)^{2} \frac{d r}{r^{1+\alpha}}\right)^{\frac{1}{2}},
$$

and also (Ste61])

$$
\left\|(-\Delta)^{\alpha / 4} f\right\|_{L^{p}\left(\mathbb{R}^{n}\right)} \leq C_{\alpha, p}\left\|D_{\alpha} f\right\|_{L^{p}\left(\mathbb{R}^{n}\right)}
$$

where

$$
D_{\alpha} f(x)=\left(\int_{\mathbb{R}^{n}} \frac{|f(x+y)-f(x)|^{2}}{|y|^{n+\alpha}} d y\right)^{\frac{1}{2}} .
$$

In CRTN01], these inequalities were extended to the setting of a unimodular Lie group endowed with a sub-laplacian $\Delta$, relying on semigroups techniques and Littlewood-Paley-Stein functionals. In particular, in [CRTN01], the authors use pointwise estimates of the kernel of the semigroup generated by $\Delta$. In the present paper, we deal with the operator $L_{M}$ for which these pointwise estimates are not available, but it turns out that $L^{2}$ off-diagonal estimates are enough for our purpose. Note that we do not obtain $L^{p}$ inequalities here. 


\section{Appendix A: Technical lemma}

We prove the following lemma.

Lemma 4.1. Let $G$ and the $x_{j}^{t}$ be as in the proof of Lemma 3.3. Then there exists a constant $\widetilde{C}>0$ with the following property: for all $\theta>1$ and all $x \in G$, there are at most $\widetilde{C} \theta^{2 \kappa}$ indexes $j$ such that $\left|x^{-1} x_{j}^{t}\right| \leq \theta \sqrt{t}$.

Proof of Lemma 4.1. The argument is very simple (see [Kan85) and we give it for the sake of completeness. Let $x \in G$ and denote

$$
I(x):=\left\{j \in \mathbb{N} ;\left|x^{-1} x_{j}^{t}\right| \leq \theta \sqrt{t}\right\} .
$$

Since, for all $j \in I(x)$

$$
B\left(x_{j}^{t}, \sqrt{t}\right) \subset B(x,(1+\theta) \sqrt{t}),
$$

and

$$
B(x, \sqrt{t}) \subset B\left(x_{j}^{t},(1+\theta) \sqrt{t}\right),
$$

one has by (1.2) and the fact that the balls $B\left(x_{j}^{t}, \sqrt{t}\right)$ are pairwise disjoint,

$$
\begin{aligned}
|I(x)| V(x, \sqrt{t}) & \leq \sum_{j \in I(x)} V\left(x_{j}^{t},(1+\theta) \sqrt{t}\right) \\
& \leq C(1+\theta)^{\kappa} \sum_{j \in I(x)} V\left(x_{j}^{t}, \sqrt{t}\right) \\
& \leq C(1+\theta)^{\kappa} V(x,(1+\theta) \sqrt{t}) \\
& \leq C(1+\theta)^{2 \kappa} V(x, \sqrt{t})
\end{aligned}
$$

and we get the desired conclusion.

\section{Appendix B: Estimates For $g_{j}^{t}$}

We prove Lemma 3.4. For all $x \in G$,

$$
\begin{aligned}
g_{0}^{j, t}(x) & =f(x)-\frac{1}{V(2 \sqrt{t})} \int_{B\left(x_{j}^{t}, 2 \sqrt{t}\right)} f(y) d y \\
& =\frac{1}{V(2 \sqrt{t})} \int_{B\left(x_{j}^{t}, 2 \sqrt{t}\right)}(f(x)-f(y)) d y .
\end{aligned}
$$

By Cauchy-Schwarz inequality and (1.1), it follows that

$$
\left|g_{0}^{j, t}(x)\right|^{2} \leq \frac{C}{V(\sqrt{t})} \int_{B\left(x_{j}^{t}, 4 \sqrt{t}\right)}|f(x)-f(y)|^{2} d y .
$$


Therefore,

$\left\|g_{0}^{j, t}\right\|_{L^{2}\left(C_{0}^{j, t}, M\right)}^{2} \leq \frac{C}{V(\sqrt{t})} \int_{B\left(x_{j}^{t}, 4 \sqrt{t}\right)} \int_{B\left(x_{j}^{t}, 4 \sqrt{t}\right)}|f(x)-f(y)|^{2} d \mu_{M}(x) d y$,

which shows Assertion A. We argue similarly for Assertion B and obtain

$\left\|g_{k}^{j, t}\right\|_{L^{2}\left(C_{k}^{j, t}, M\right)}^{2} \leq \frac{C}{V\left(2^{k} \sqrt{t}\right)} \int_{x \in B\left(x_{j}^{t}, 2^{k+2} \sqrt{t}\right)} \int_{y \in B\left(x_{j}^{t}, 2^{k+2} \sqrt{t}\right)}|f(x)-f(y)|^{2} d \mu_{M}(x) d y$,

which ends the proof.

\section{REFERENCES}

$\left[\mathrm{AHL}^{+}\right.$02] P. Auscher, S. Hofmann, M. Lacey, A. McIntosh, and P. Tchamitchian. The solution of the Kato square root problem for second order elliptic operators on $\mathbb{R}^{n}$. Ann. of Math. (2), 156(2):633-654, 2002.

[BBCG08] D. Bakry, F. Barthe, P. Cattiaux, and A. Guillin. A simple proof of the Poincaré inequality for a large class of probability measures including the log-concave case. Electron. Commun. Probab, 13:60-66, 2008.

[CRTN01] T. Coulhon, E. Russ, and V. Tardivel-Nachef. Sobolev algebras on Lie groups and Riemannian manifolds. Amer. J. Math., 123:283-342, 2001.

[Dav80] E.B. Davies. One-parameter semigroups, volume 15 of London Mathematical Society Monographs. Academic Press, Inc., London-New York, second edition, 1980.

[Gaf59] M.P. Gaffney. The conservation property of the heat equation on Riemannian manifolds. Comm. Pure Appl. Math., 12:1-11, 1959.

[Gui73] Y. Guivarc'h. Croissance polynomiale et période des fonctions harmoniques. Bull. Soc. Math. France, 101:333-379, 1973.

[HK00] P. Hajłasz and P. Koskela. Sobolev met Poincaré, volume 145 (688) of Mem. Amer. Math. Soc. Amer. Math. Soc., 2000.

[Jer86] D. Jerison. The Poincaré inequality for vector fields satisfying Hörmander's condition. Duke Math. J., 53(2):503-523, 1986.

[Kan85] M. Kanai. Rough isometries and combinatorial approximations of geometries of noncompact riemannian manifolds. J. Math. Soc. Japan, 37:391-413, 1985 .

[MRS09] C. Mouhot, E. Russ, and Y. Sire. Fractional poincaré inequalities for general measures. http://arxiv.org/abs/0911.4563, 2009.

[NSW85] A. Nagel, E. M. Stein, and S. Wainger. Balls and metrics defined by vector fields i: Basic properties. Acta Math., 155:103-147, 1985.

[SC95] L. Saloff-Coste. Parabolic Harnack inequality for divergence form second order differential operators. Potential Analysis, 4(4):429-467, 1995.

[Ste61] E.M. Stein. The characterization of functions arising as potentials I. Bull. Amer. Math. Soc., 67:102-104, 1961.

[Str67] R.S. Strichartz. Multipliers on fractional Sobolev spaces. J. Math. Mech., 16:1031-1060, 1967.

[Var87] N.T. Varopoulos. Fonctions harmoniques sur les groupes de Lie. C. R. Acad. Sci. Paris Ser. I Math, 304(17):519-521, 1987. 
Emmanuel Russ- Université Paul Cézanne, LATP, Faculté des Sciences et Techniques, Case cour A

Avenue Escadrille Normandie-Niemen, F-13397 Marseille, Cedex 20, France et

CNRS, LATP, CMI, 39 rue F. Joliot-Curie, F-13453 Marseille Cedex 13, France

Yannick Sire- Université Paul Cézanne, LATP, Faculté des Sciences et Techniques, Case cour A

Avenue Escadrille Normandie-Niemen, F-13397 Marseille, Cedex 20, France et

CNRS, LATP, CMI, 39 rue F. Joliot-Curie, F-13453 Marseille Cedex 13, France. 